\title{
Bronchial thermoplasty: implementing best practice in the era of cost containment
}

This article was published in the following Dove Press journal:

Journal of Asthma and Allergy

26 July 2017

Number of times this article has been viewed

\author{
Laren D Tan' \\ Nicholas Kenyon ${ }^{2}$ \\ Ken Y Yoneda ${ }^{2}$ \\ Samuel Louie ${ }^{2}$ \\ 'Division of Pulmonary, Critical Care, \\ Hyperbaric and Sleep Medicine, \\ Department of Internal Medicine, \\ School of Medicine, Loma Linda \\ University, Loma Linda, CA, USA; \\ ${ }^{2}$ Division of Pulmonary, Critical Care \\ and Sleep Medicine, Department of \\ Internal Medicine, School of Medicine, \\ University of California, Davis, \\ Sacramento, USA
}

Abstract: Increasing dependence on advanced technologies in the 21 st century has created a dilemma between the practice and business of medicine. From information technology to robotic surgery, new technologies have expanded treatment possibilities and have potentially improved patient outcomes and safety. Simultaneously, their escalating costs limit access for certain patients and health care facilities. Nevertheless, medical decisions should not simply be based on cost. Input from physicians and other health care specialists as well as adherence to best practice position statements, are vital to implementing truly cost-effective strategies in medicine. Bronchial thermoplasty (BT), a US Food and Drug Administration approved bronchoscopy procedure in difficult-to-control persistent asthma, is a prime example of a new technology facing cost and implementation challenges. We discuss the specific indications and contraindications for BT and review recent real-world experiences that can provide the foundation for building a comprehensive asthma program that provides BT for difficult-to-control asthma patients who fail national guideline treatment recommendations after an adequate clinical trial of one. We also offer insight into the barriers to implementing a successful BT program and strategies for overcoming them.

Keywords: asthma, severe asthma, severe refractory asthma, biologic resistant asthma, BT

\section{Introduction}

Historians will debate the pros and cons of incorporating information technologies and computer science into the practice and business of medicine in the 21 st century. The proliferation, assimilation and dependence on new technologies affect all disciplines in medicine. Implementation of such technological advances should be based on a careful analysis of cost-benefit ratios and safety. Few technological interventions are as controversial as bronchial thermoplasty (BT), despite its proven efficacy, safety and clinical effectiveness. We review and discuss BT best practice key points with the aim of: 1) fostering adoption by institutional leadership and 2) unifying BT skeptics and advocates in the health care industry. In addition, we provide strategies for increasing asthma awareness in the public and medical communities by leveraging various facets of traditional as well as social media to ensure that patients and providers have legitimate access to BT.

Division of Pulmonary, Critical Care,

Hyperbaric and Sleep Medicine,

Department of Internal Medicine, School of Medicine, Loma Linda University, II 234 Anderson Street, Room 6433, Loma Linda, CA 92354, USA

$\mathrm{Tel}+\mathrm{I} 9095584489$

Fax + I 909558058 I

Email latan@llu.edu

\section{Implementing a successful BT program}

Three important areas should be the focus of any effort to bring BT to institutions and patients: 1) the alignment of clinical leadership and providers with current best practice recommendations to improve patient outcomes and safety, 2) lobbying local clinical 
champions to protect provider and patient choice in treatment options, and 3) leveraging media to raise public awareness of advances in chronic disease management (including new therapeutics). All 3 areas are vital for patients and health care teams to reap the safety and benefits of clinical practice and experience in the era of cost containment (Figure 1).

\section{Alignment of clinical leadership and providers}

As technology grows at an accelerated rate, the medical community struggles to keep pace in the areas of electronic medical documentation and medical therapeutics. Although new medical devices and smart phone applications are welcomed by those who can adapt easily to technology, others resist these innovations. Unfortunately, the adoption of such a technology, for example, BT for the treatment of severe asthmatics, can be a difficult and frustrating experience to both patient and provider, particularly when there is misalignment of views between leadership within the hospital and health care providers, including pulmonologists and allergists. Unifying these views requires being an asthma patient advocate and having an appreciation of the dangers to asthmatics who continue to live a poor quality of life with frequent exacerbations despite adherence to conventional pharmacotherapies, allergy shots, removal of asthma triggers (i.e., tobacco smoke, dust, gastroesophageal reflux disease), assurance of good inhaler technique and compliance to prescribed inhaler medications. For some, scientific rationale, clinical trials and/or best practice guidelines are a sufficient basis for change. For others, only the

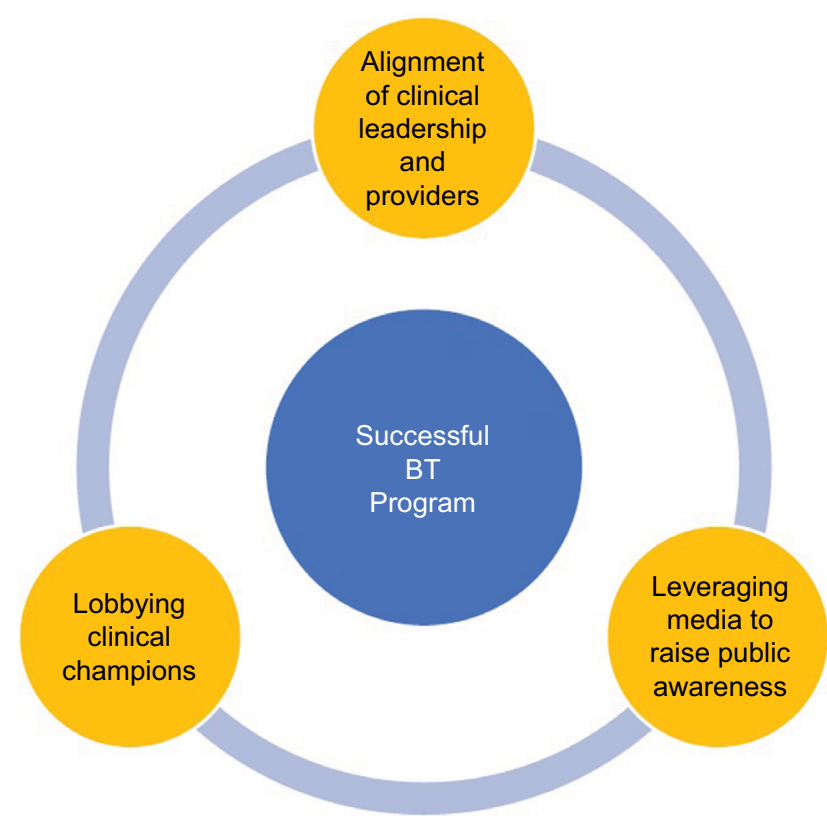

Figure I Key elements surrounding a successful BT program. Abbreviation: BT, bronchial thermoplasty. objective improvement of their personal patients will turn their minds toward acceptance of BT as a viable treatment strategy.

Resistance to BT arise from not fully understanding how BT benefits asthma patients at the pathophysiological level. While first described in 1922 by Hubert and Koessler in cases of fatal asthma, airway remodeling exists in varying degrees of asthma severity and in both the small and large airways. ${ }^{1}$ Airway remodeling refers to the structural changes in the airways of the asthmatic subjects that are not typically present in healthy subjects. Structural changes include the loss of epithelial integrity, thickening of the basement membrane, subepithelial fibrosis, goblet cell and submucosal gland enlargement, increased smooth muscle mass, decreased cartilage integrity and increased airway vascularity. ${ }^{1-3}$ Such changes are primarily a result of ongoing chronic inflammation that involves multiple inflammatory pathways, including CD4+ T-cells, interleukins (IL-4, IL-5, IL-13), eosinophils, neutrophils and mast cells. ${ }^{4}$ Other factors include the duration of uncontrolled asthma, (often associated with reduced lung function), increased airway hyperresponsiveness (AHR) and asthma symptoms, as well as increased utilization of asthma therapy medications.$^{5-7} \mathrm{BT}$ significantly changes pathologic airway remodeling by decreasing the hypertrophied smooth muscle mass, airway inflammation, neuroendocrine epithelial cells and bronchial nerve endings. It improves asthma symptom control ostensibly by decreasing bronchospasm and AHR. ${ }^{8,9}$ (Figure 2A and B, biopsy specimens taken before and 3 months after BT; note that Figure 2B, rather than representing the average, exemplifies a case in which smooth muscle was virtually absent). ${ }^{9}$

As a validated adjunct to the armamentarium of available asthma therapy, BT provides hope for the $5 \%-10 \%$ defined as severe asthmatics refractory to conventional therapy. ${ }^{10-12}$ Still largely thought to be innovative, BT debuted in 2010 when it was approved by the US Food and Drug Administration
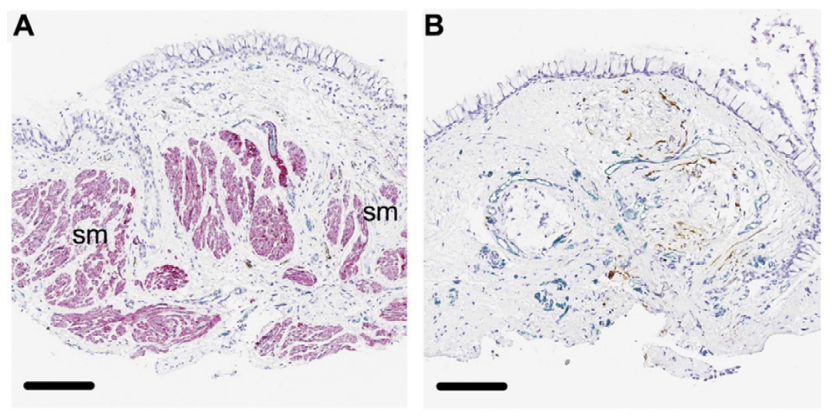

Figure 2 Structural effects of BT in bronchial biopsy specimens from patients with severe asthma.

Notes: (A) Specimen taken before BT. (B) Specimen taken 3 months after BT (this image, rather than representing the average, exemplifies a case in which smooth muscle was virtually absent). Scale bars $=250 \mu \mathrm{m}$.

Abbreviations: BT, bronchial thermoplasty; sm, smooth muscle. 
(FDA). ${ }^{11}$ A multi-centered, randomized, sham bronchoscopy controlled trial by Castro et al demonstrated that BT improved asthma symptom score based on the Asthma Quality of Life Questionnaire and other secondary end points (percentage of symptom-free days, total symptom core, forced expiratory volume 1 second, peak expiratory flow, rescue medication use) at 1 year. ${ }^{11,13}$ A Cochrane review on BT for moderate or severe persistent asthma in adults found that BT provided a modest clinical benefit in quality of life and lower rates of asthma exacerbation, but no significant difference in asthma control scores. ${ }^{14}$ Careful follow-up of $>85 \%$ of the BT group out to 5 years demonstrated that the reduction in severe exacerbation seen in year 1 was maintained with no safety concerns. ${ }^{12}$ The paucity of BT programs available to asthma patients in the USA is evidence of the misalignment of views as to the safety and efficacy of BT in the real world. Cost-mediated health care decisions by hospital administrators and insurance companies often discount the needs of the individual asthmatic and the long-term benefits to all patients over time, including the reduction in need for controller medications and a decrease in the frequency of acute exacerbations. Commonly held views by asthmatologists include the perception that BT does not affect airway inflammation and that only bronchodilators have any effect on the target tissue for BT: bronchial smooth muscle. Leaving difficultto-control patients on life-long biologics, prednisone and/or high-dose inhaled corticosteroids is neither completely safe nor financially and medically justifiable if the patient remains not well controlled or very poorly controlled.

The treatment of all asthma patients should be supported by the most convincing available evidence of safety and efficacy. BT is not a cure for asthma but it is safe and effective in enhancing control over asthma symptoms and acute exacerbations. BT is advocated for the treatment of severe asthma by the American College of Chest Physicians (CHEST), Asthma and Allergy Foundation of America (AAFA), American College of Asthma, Allergy and Immunology (ACCAI), British Thoracic Society (BTS), and the Global Initiative for Asthma (GINA), which is an international consensus program that presents recommendations for comprehensive asthma management strategies. ${ }^{15-19}$ The United States National Institutes of Health National Asthma Education and Prevention Program guidelines for clinical practice guidelines have not been updated since 2007, before BT was FDA-approved.

Nevertheless, the role of BT continues to be debated by the medical asthma community and insurance companies continue to deny authorization of payment. Aligning clinical leaders and providers of asthma treatment on the understanding of the: 1) pathophysiology of asthma; 2) effect of BT on airway remodeling; 3 ) clinical trial data supporting BT; and 4) medical professional societies supporting its use, will help BT to gain greater acceptance and help administrators and insurance companies who hold overwhelming financial and political powers in health care resource decision-making to invest in BT.

\section{Lobbying clinical champions}

All health care decisions should be patient-centered. However, it is apparent that all health care decisions rely heavily on the cost-mediated factors. Adoption of BT at any institution can be arduous, but offsetting the hard work are patient outcomes that can be extremely rewarding. Clinical champions are key to overcoming barriers and establishing a successful BT program. Within each group or division lies a political agenda, financial barriers, personal bias, organizational agendas, and reluctance to change. Being familiar with the organizational arena and tone provides further insight on how to create a successful program. Lobbying key thought leaders about BT and recruiting clinical champions is vital in promoting further support. Prior to fostering awareness, identification of community need for BT must also be considered. Asthma prevalence was reported to have increased from $7.3 \%$ in 2001 to $7.7 \%$ for $2014 .{ }^{20}$ Of the 25 million asthmatics, $5 \%-10 \%(\sim 1.2-2.4$ million $)$ have been reported to have severe persistent asthma, asthma that is refractory to available standard oral and inhaled therapies. ${ }^{5}$ Although the needs for BT in a community may not on the surface appear to reflect the national prevalence, the magnitude of the problem may very well be unmasked with BT awareness. Within 8 months of starting our BT program at Loma Linda University, we have become the referral site for 3 neighboring hospitals and surrounding clinics. In addition, we have provided a validated therapeutic option to the asthma treatment pathway for our pediatric asthma colleagues for when their patients turn 18. In the Northern California Central Valley, UC Davis remains the only university-based center for BT utilizing a patient care-centered pathway for referrals (Figure 3).

As is true for most new technologies, upfront and ongoing financial expenditures concern all health care administrators, insurers, patients and health care providers. Establishing a BT program requires lobbying clinical champions to inform all stakeholders that business models for BT have demonstrated it to be cost-effective and potentially cost-saving. ${ }^{21,22}$ Most importantly, BT is a vital alternative in asthma patientcentered care. 


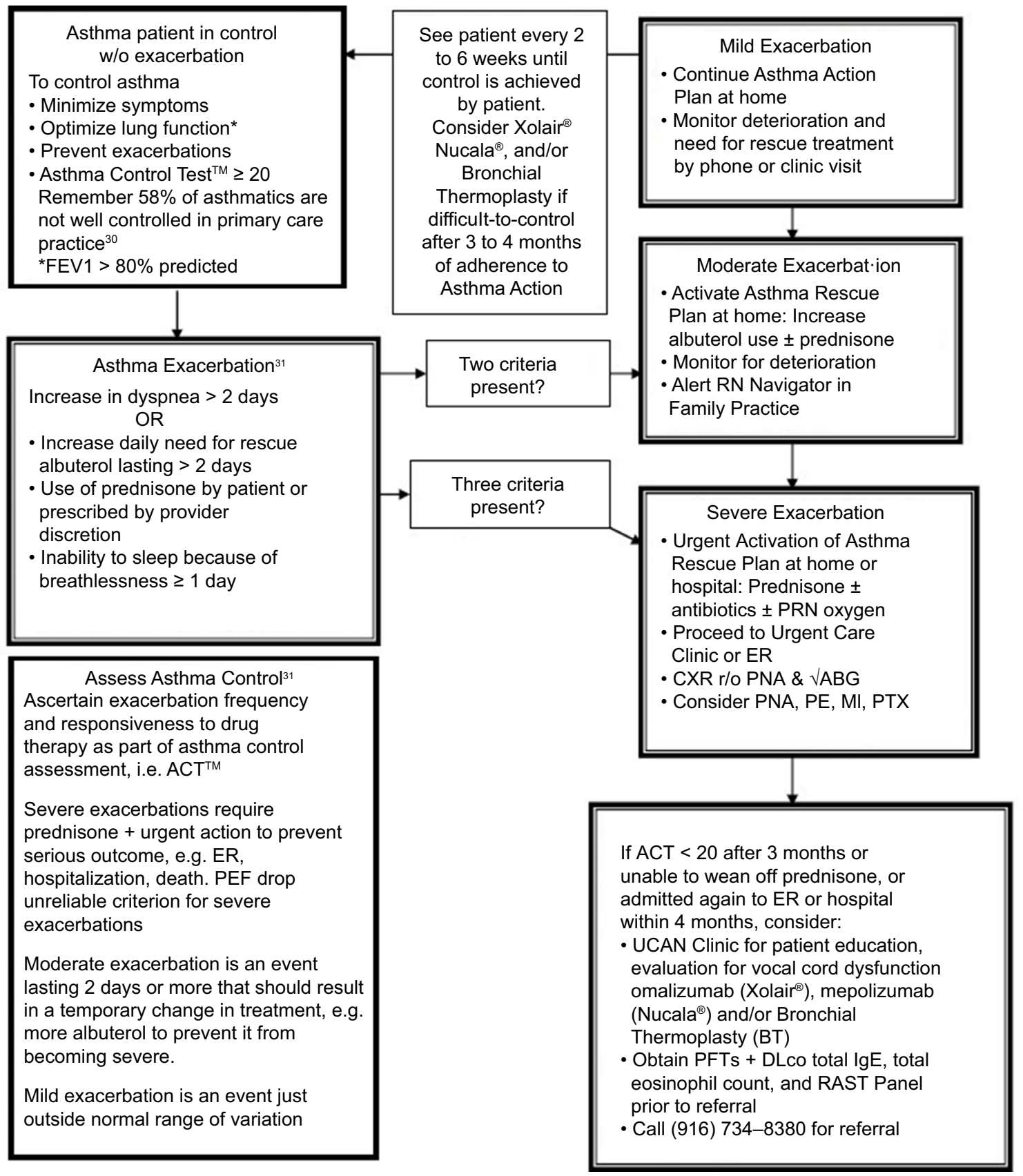

Figure 3 UC Davis Health System ROAD ${ }^{\mathrm{TM}}$ Center Asthma Exacerbation and UCAN Referral Algorithm.

Note: Data from Mintz et al ${ }^{30}$ and Reddel et al. ${ }^{31}$

Abbreviations: $\sqrt{ }$, to check; $A B G$, arterial blood gas; $A C T$, asthma control test; $B T$, bronchial thermoplasty; CHF, congestive heart failure; CXR, chest X-ray; DLco, diffusing capacity of the lungs for carbon monoxide; ER, emergency room; FEVI, forced expiratory volume I second; IgE, immunoglobulin E; MI, myocardial infarction; PE, pulmonary embolism; PEF, peak expiratory flow; PFT, pulmonary function test; PNA, pneumonia; PRN, as needed; PTX, pneumothorax; r/o, rule out; RAST, radioallergosorbent test; RN, registered nurse; ROAD, reversible obstructive airway disease; UC, University of California; UCAN, University of California Davis Asthma Network; w/o, without.

BT requires the individual clinician to depart from a linear and traditional mode of treatment and consider a therapy that some view as disruptive. It was "out-of-the-box," forward thinking that made it possible to provide BT as an additional modality for asthmatics who have failed conventional therapy. Frequent and regular group discussions of plan of care for the most pressing and refractory asthmatic cases in a practice setting can foster thought-provoking discussions, which may highlight the need for stopping or beginning therapy that are outside the usual asthma regiment. Sharing our experience and some of our most remarkable BT cases can provide the incentive to accept change during a clinical trial of one.

Recognizing the national and community needs, monetary advantages and acquiescence to progressive asthma therapies provide insufficient inertia unless a consistent force of "passion" is applied to fuel the drive in providing hope 
to severe asthmatics. It is the instinct of competence, trust, emotional awareness, integrity, open communication and reliability that coalesce to form a passion to construct and sustain a successful BT program. Such attributes, in addition to life changing patient-physician case studies ${ }^{23}$ of post-BT patients, assist in persuading skeptical minds in favor of BT and other pulmonary therapies.

\section{Leveraging media to raise public awareness}

Leveraging asthma awareness utilizing social media platforms as well as media (television, newspapers, radio, internet) provides a broad communication platform for health care professionals to reach patients within the community. This almost ubiquitous presence allows one to reach patients on their terms. Social media has altered the landscape of modern medicine ${ }^{24}$ but has also provided a means of transparency and connection, which is particularly germane in the realm of asthma therapy. The community became aware of our asthma clinic at Loma Linda University through medical education segments we provided to local television news agencies,${ }^{25}$ through clinical insight to newspaper agencies ${ }^{26}$ and most recently utilizing Facebook live chat to discuss asthma and its affiliated therapies. Through this platform, we promoted asthma awareness to over 17,000 people with 6200 video views.

The portmanteau glocal (global and local), epitomizes our thoughts and efforts regarding asthma management and treatments. Circumspection back to the basics of asthma prompts review that asthma develops through the interaction of genetic factors with environmental exposures. Strong evidence has linked exposure to allergens commonly found in homes, such as those derived from dust mites, cockroaches, rodents, molds, and pet dander, to sensitization and subsequent asthma incidence and morbidity. In addition to allergens, other indoor asthma triggers include tobacco smoke, nitrogen oxides from combustion devices, and irritants from volatile organic compounds and fungi. ${ }^{27}$ Many patients in our communities in Southern California and Northern California do not have the luxury to move and avoid some of the most common allergens known to exacerbate asthma. Others have companion pets and would rather experience a near-death asthma exacerbation than abstaining from being around them. Air pollution is a contributing culprit and for decades has been associated with asthma exacerbations, emergency room visits, transitory reductions in lung function, and respiratory symptoms. ${ }^{27,28}$ Allergic responses have also been associated with pollutant exposure. While several pollutants have been associated with these outcomes, particulate air pollution has been extremely problematic. ${ }^{28}$ California was most recently in its sixth year of severe drought, the state also experienced multiple record-shattering heat waves. The combination of dry foliage with extreme dry heat has led to an increase in airborne particulate matter from uncontrolled wildfires, and together with other sources of pollutants (such as emissions from cars and factories) has created a seasonal ozone that can be caustic to any lung. ${ }^{26}$ Understanding and anticipating global trends in temperature, weather, ozone and air particulate levels can better assist in attending the cyclical community needs in asthma treatment, and in some cases, explicates a patient's continuous and severe refractory asthma symptoms. We anticipate that BT will be of greatest value to asthma patients residing in cities where environmental challenges are the greatest. At present, only a failure to control asthma symptoms and risks with conventional pharmacotherapy identify candidates for BT.

\section{Discussion and conclusion}

Increasingly, hospitals are leveraging formal technology appraisals and evidence review processes prior to the purchase of new technologies and onboarding of new services. However, while these reviews will ensure an open-eyed understanding of the technology and service, it is insufficient to ensure success. Best practice success should also be weighted on the community impact for treatment of poorly controlled severe, persistent asthma utilizing BT and its potential to reduce both health care resources and financial burden. ${ }^{21,22,29}$ Although the practicing allergist and pulmonologists can do much to ensure success post-approval, the 3 key strategies described above (alignment of clinical leadership and providers, lobbying local clinical champions and leveraging media to raise public awareness) can exponentially enhance the likelihood of success.

\section{Disclosure}

KY Yoneda is a paid consultant for Boston Scientific. The other authors report no conflicts of interest in this work.

\section{References}

1. Bergeron C, Tulic MK, Hamid Q. Airway remodelling in asthma: from benchside to clinical practice. Can Respir J. 2010;17(4):e85-e93.

2. Sumi Y, Hamid Q. Airway remodeling in asthma. Allergol Int 2007;56(4):341-348.

3. Woodruff PG, Fahy JV. Airway remodeling in asthma. Semin Respir Crit Care Med. 2002;23(4):361-367.

4. Chung KF. Targeting the interleukin pathway in the treatment of asthma Lancet. 2015;386(9998):1086-1096.

5. Tan LD, Bratt JM, Godor D, Louie S, Kenyon NJ. Benralizumab: a unique IL-5 inhibitor for severe asthma. JAsthma Allergy. 2016;9:71-81. 
6. Tan LD, Chan AL, Albertson TE. New combination treatments in the management of asthma: focus on fluticasone/vilanterol. J Asthma Allergy. 2014;7:77-83.

7. O’Byrne PM, Naji N, Gauvreau GM. Severe asthma: future treatments. Clin Exp Allergy. 2012;42(5):706-711.

8. Denner DR, Doeing DC, Hogarth DK, Dugan K, Naureckas ET, White SR. Airway Inflammation after Bronchial Thermoplasty for Severe Asthma. Ann Am Thorac Soc. 2015;12(9):1302-1309.

9. Pretolani M, Bergqvist A, Thabut G, et al. Effectiveness of bronchial thermoplasty in patients with severe refractory asthma: clinical and histopathologic correlations. J Allergy Clin Immunol. 2016;139(4):1176-1185.

10. Castro M, Rubin A, Laviolette M, et al. Persistence of effectiveness of bronchial thermoplasty in patients with severe asthma. Ann Allergy Asthma Immunol. 2011;107(1):65-70.

11. Castro M, Rubin AS, Laviolette M, et al. Effectiveness and safety of bronchial thermoplasty in the treatment of severe asthma: a multicenter, randomized, double-blind, sham-controlled clinical trial. Am J Respir Crit Care Med. 2010;181(2):116-124.

12. Wechsler ME, Laviolette M, Rubin AS, et al. Bronchial thermoplasty: long-term safety and effectiveness in patients with severe persistent asthma. J Allergy Clin Immunol. 2013;132(6):1295-1302.

13. Thomson NC, Rubin AS, Niven RM, et al. Long-term ( 5 year) safety of bronchial thermoplasty: asthma Intervention Research (AIR) trial. BMC Pulm Med. 2011;11:8.

14. Torrego A, Sola I, Munoz AM, et al. Bronchial thermoplasty for moderate or severe persistent asthma in adults. Cochrane Database Syst Rev. 2014(3):CD009910.

15. The American College of Chest Physicians (CHEST). Position statement for coverage and payment for bronchial thermoplasty; 2014. Available from: http://www.chestnet.org/News/CHESTNews/2014/05/PositionStatement-for-Coverage-and-Payment-for-Bronchial-Thermoplasty. Accessed May 12, 2014

16. American College of Allergy, Asthma and Immunology (ACCAI). Statement on bronchial thermoplasty; 2015. Available from: http:// college.acaai.org/publications/advocacy-insider/statement-bronchialthermoplasty. Accessed January 1, 2015.

17. INTERASMA, the Global Asthma Association. Bronchial thermoplasty: an additional option for managing patients with severe asthma; 2014. Available from: http://www.interasma.org/images/manifesto3.pdf. Accessed October 31, 2014.

18. Global Initiative for Asthma (GINA). Global strategy for asthma management and prevention; updated 2015. Available from: http:// ginasthma.org/archived-reports/. Accessed May 19, 2015.
19. British Thoracic Society (BTS). British guideline on the management of asthma; 2014. Available from: https://www.brit-thoracic.org.uk. Accessed October 31, 2014

20. Centers for Disease Control and Prevention (CDC). Most recent asthma data; 2017. Available at: https://www.cdc.gov/asthma/most_recent_data. htm. Accessed February 1, 2017.

21. Zein JG, Menegay MC, Singer ME, et al. Cost effectiveness of bronchial thermoplasty in patients with severe uncontrolled asthma. J Asthma. 2016;53(2):194-200.

22. Zafari Z, Sadatsafavi M, Marra CA, Chen W, FitzGerald JM. Costeffectiveness of bronchial thermoplasty, omalizumab, and standard therapy for moderate-to-severe allergic asthma. PLoS One. 2016;11(1): e0146003.

23. Pearson SD, Raeke LH. Patients' trust in physicians: many theories, few measures, and little data. J Gen Intern Med. 2000;15(7): $509-513$.

24. George DR, Rovniak LS, Kraschnewski JL. Dangers and opportunities for social media in medicine. Clin Obstet Gynecol. 2013;56(3): 453-462.

25. Tan L. Asthma and bronchial thermoplasty; 2016. Available from: http://www.kesq.com/marketplace/loma-linda/dr-laren-tan-md-deptpulmonary-critical-care-medicine-1/128235698. Accessed October 20, 2016.

26. Jablon R. Southern California having smoggiest summer since 2009; 2016. Available at: http://bigstory.ap.org/article/1bedc06c4eae40658af $596329392 \mathrm{~d} 32 \mathrm{e} /$ southern-california-having-smoggiest-summer-2009. Accessed August 12, 2016.

27. Krieger J. Home is where the triggers are: increasing asthma control by improving the home environment. Pediatr Allergy Immunol Pulmonol. 2010;23(2):139-145.

28. Sofer T, Baccarelli A, Cantone L, et al. Exposure to airborne particulate matter is associated with methylation pattern in the asthma pathway. Epigenomics. 2013;5(2):147-154.

29. Cangelosi MJ, Ortendahl JD, Meckley LM, et al. Cost-effectiveness of bronchial thermoplasty in commercially-insured patients with poorly controlled, severe, persistent asthma. Expert Rev Pharmacoecon Outcomes Res. 2015;15(2):357-364.

30. Mintz M, Gilsenan AW, Bui CL, et al. Assessment of asthma control in primary care. Curr Med Res Opin. 2009;25(10):2523-2531.

31. Reddel HK, Taylor DR, Bateman ED, et al. An official American Thoracic Society/European Respiratory Society statement: asthma control and exacerbations: standardizing endpoints for clinical asthma trials and clinical practice. Am J Respir Crit Care Med. 2009;180(1): 59-99.
Journal of Asthma and Allergy

\section{Publish your work in this journal}

The Journal of Asthma and Allergy is an international, peer-reviewed open access journal publishing original research, reports, editorials and commentaries on the following topics: Asthma; Pulmonary physiology; Asthma related clinical health; Clinical immunology and the immunological basis of disease; Pharmacological interventions and

\section{Dovepress}

new therapies. This journal is included in PubMed. The manuscript management system is completely online and includes a very quick and fair peer-review system, which is all easy to use. Visit http://www. dovepress.com/testimonials.php to read real quotes from published authors. 